\title{
Somatic senses required for the emotional design of upper limb prosthesis
}

\author{
Luisa M. Arruda ${ }^{1}$, Luís F. Silva ${ }^{2}$, Helder Carvalho ${ }^{1}$, Miguel A. F. Carvalho ${ }^{1}$, and Fer- \\ nando B. N. Ferreira ${ }^{1}$
${ }^{1}$ 2C2T - Centro de Ciência e Tecnologia Têxtil, University of Minho, Guimarães, Portugal
${ }^{2}$ MEtRICs - Mechanical Engineering and Resource Sustainability Center, University of Minho, Guimarães, Portugal luisamendesarruda@gmail.com

\begin{abstract}
Despite the technological advances associated with prostheses, the total embodiment is still the great challenge in the segment of assistive technology. One of the main aspects is that the bionic member's sensibility is not responsive to the environment that surrounds it. The purpose of this study aims to identify the perceptual modalities of the somatic senses that are required for a more sensible prosthesis. The methodological strategy contemplates literary research and proposes an interrelationship between neuroscience with philosophi$\mathrm{cal} /$ cultural studies, which regards the different concepts of sensory experience. Such data were analyzed quantitatively and qualitatively. The main conclusion points out that it would be important to unite the nine physiological requirements identified in the state of the art, with the ontological image construction of the prosthesis, in order to create a sensory experience that, in addition to the perceptive organs, builds up by the visual areas of the brain.
\end{abstract}

Keywords: Emotional design $\cdot$ Upper limb prosthesis $\cdot$ Sensibility $\cdot$ Somatic sense

\section{Introduction}

The practice of limb amputation arose in the Neolithic period, 43000 B.C. [1] and the reasons for such an act were punitive, therapeutic, and ritualistic. Subsequent to the amputation procedure, the prostheses were developed to meet the requirements of functionality, aesthetic appearance and a psycho-spiritual sense of totality, around 1500 B.C. [2]. However, it is with the advent of the two great world wars that prostheses became modernized regarding their shape. In the 21 st century, advances in additive manufacturing have allowed such devices to be more accessible, customizable and made from both flexible and rigid raw materials [3]; and the progress of nanotechnology, with the miniaturization of electronic devices - especially sensors and actuators - have added functionalities to the prostheses identified as degrees of freedom (DOF), which in turn have been allowing the amputee a greater diversity of ac- 
tivities of daily living (ADL), such as undoing buttons, simulating food cuts, moving an empty can, among others.

Although the mechanical and electrical advancements of prosthetic devices are irrefutable, upon asking a man with transhumeral amputation - who tested a technological prosthesis - what he considered to be improved on his artificial limb, he replied that he would like to hold a loved one's hand and really feel it, beyond the mechanical grasping movement [4]. In other words, the sensory character related to the functionality of prostheses as the crucial point to be developed by the assistive technology segment is evidenced.

The fact is that the act of touching a loved one's hand is a communicational action constructed in otherness, which means that such action does not generate fixed sensations; instead, they vary from enunciator to enunciator. In its turn, individual reactions go beyond the dimension of the somatic senses and express a combination of psychophysical mechanisms. Moreover, such combinations externalize codes that make possible the interpretation of affection by each enunciator of the communicational act (amputated or not).

In this sense, in order to enumerate assumptions for the design and development of a prosthesis that meets the diligence in question, this study proposes to investigate the sensibility concepts present in the current prostheses and interpose through an ontological approach of the sensory experience.

\section{Methodology}

The research strategy adopted in this paper is the exploratory study since one intends to enlighten the phenomenon in question - here referred to as the sensibility in prostheses - to conceive research through diverse techniques that involves bibliographical survey and exploration of patents. This methodology has the objective of defining the problem in question to then raise the somatosensory requirements required for the design and development of more emotional upper limb prosthesis.

Patent research was carried out using Derwent Innovation software (Clarivate Analytics). The keywords used were: upper limb prosthesis; bionic prosthetic and bionic limb. In turn, the sub-words searched were: tactile feedback; sensibility; sensing; sensation and affectivity.

Simultaneously, a bibliographical revision was made on the term "sensibility", aiming to define it according to a philosophical approach, specifically by the perspective of the Italian author Emanuele Coccia [5]. This author was evidenced as he deals with sensibility through the ontology of the images, dialoguing on an anatomical and prosthetic body and explores Fashion as a form of construction of the images and, therefore, of the sensibility in an autonomous sphere. Thus, Coccia contrasts essential concepts that allow us to elucidate about the prosthesis, beyond the medical devices, and closer to the emotional design. 


\section{Results}

The patent search found 24 results, however, only 5 refer directly to sensibility. The first patent aimed at proposing sensory reconfiguration dates back to 1998. It distinguishes itself by being a method and device that creates tactile sensations through sensors and sends them to the individual. Such sensations relate to the texture and sliding of objects in contact with the prosthetic hand [6]. In addition, a system and method are cited for providing a sensation in a member with a prosthetic device or sensory deficiency: sensors positioned at the foot of the prosthesis contact the sock and produce a vibration stimulus in the residual limb, which is aided by an electrical unit to adjust and control the stimulus [7]; a mechanism and method for designing and constructing a mechanical interface between a wearable device and a segment of the human body [8]; and sensors that can maintain a comfortable temperature and humidity between the device and the residual limb of the individual [9].

In addition to these, another patent was also analyzed concerning a method and system to promote proprioceptive feedback, a characteristic present in the somatic system [10]. The others were discarded given that they do not fit the sensory system, or they refer to invasive procedures: 12 are directed to the identification and control of movements of the prosthetic limb; 1 refers to the detection of the error of humanmachine interaction; 1 relates to a hospital control system; and 5 correspond to neuromuscular interference models.

\section{Discussion}

The somatosensory system allows the human being to obtain sensitive experiences with the environment that surrounds him, namely touch, pain, temperature and proprioception. Unlike other sensory systems present in the body, such as taste, smell, sight and hearing, the somatic system has receptors on distinct parts of the body.

Communication begins from contact with the outside world. The skin of the upper limb receives stimuli that are translated into neural signals. This information goes to the central nervous system and the brain realizes their perception. This means that the characteristics of the stimulus - such as intensity, duration, position and, sometimes, direction - are encoded by the sensory receptor. In turn, the central nervous system processes the activity of the receptors and uses this data to generate coherent perceptions. From here the brain uses sensory information to plan and coordinate movements [11].

As the research presented, $50 \%$ of the patents are directed to mimic the motor system of the human body. Concerning somatosensory awareness, only about $20 \%$ seek to achieve sensory mechanisms, including techniques such as sensory reeducation, tactile kinesthetic orientation, repetitive sensory practice or desensitization.

Concerning the sensory reeducation, the literature review pointed out that sensations are mimicked by detectors of temperature, texture, slip, vibration, pressure, torsion, impedance and position. Therefore, by the aspect of medicine, such survey pointed out that these nine characteristics are the desirable requirements for a sensory prosthesis. 
However, human sensations have more than physiological components. For philosophy, for example, man survives through sensations, and the senses are what the individual trusts and depends on. Therefore, the interdependence of the experience of the sensible to the living human condition is noticed. However, unlike the medical approach, the sensitive ontology reports that the production of sensibility is not related to contact with the perceptual organ, on the contrary, it is emphasized that sensibility is beyond. This approach describes the sensible as being the image in the broad sense, and therefore, it is precisely through the ability to produce images that man is affected by the experience of sensibility. Moreover, the ontological idea of image is the representation of something out of its place, in an independent sphere. In other words, philosophy approaches the science of the sensitive and ratifies that the sensation is not caused by the perceptive organs but by the construction of images in our brain [5].

Not surprisingly, recent studies on the neurological representation of artificial limbs in amputees revealed that the more the individual is subjected to the daily use of prosthetics, the more visual areas of the brain in the lateral occipitotemporal cortex reorder and begin to respond to the prostheses' images [12]. This has been demonstrated by the increased connectivity between the visual and sensory-motor areas of the brain linked to the functionality of the hands. In addition, the same study revealed that the triad: activities performed by the bionic member; visual-motor connectivity; and frequent use of the prosthesis in daily activities can progressively cause the brain to reuse neural resources originally developed, from the visual representation, to reshape the motor system in order to control the prostheses. In other words, to live the experience of the sensitive, there is in both approaches (philosophical and neurological) the construction of images in the brain as a way of relating sensory stimuli to medical devices.

Philosophy describes that phenomenon such as dreams, fashion, and language are capable of producing images, and complements that when an object becomes a phenomenon, there is sensation. Therefore, if the prosthesis has not yet reached the degree of embodiment, it can be identified as an object external to the body. Then, the question arises: how can the prosthesis stop being an external object and, through the emotional design, approach the embodiment integrated into the anatomic body?

Through the discursive reasoning presented, it is worth thinking about new measures/materials/forms for the design and development of a prosthesis that approaches more of a wearable piece - integral to the corporeal visuality of the individual - than just a medical device. In addition to the related physiological requirements for the development of an "emotional" prosthesis, it is necessary for its design to avoid attempting to replace the missing limb as a replica of what it was, since such a point only evidences the loss. It is up to the designer to identify the spirit that singularly inhabits each individual in order to materialize a wearable piece/limb, which expresses such language. In addition, it is intended that such materialization will be at the same time the construction of an orthopedic image of all the limbs, through a skin constructed with unconventional materials and forms, beyond the design of the anatomical body. 


\section{Conclusions}

For philosophy, the living one is, above all, the one who is able to give a sensible existence of that which dwells within him. This is because the body, if observed by the one who lives with it, is never a figure, a form, but a series of sensory states that interpenetrate themselves. In this sense, this paper proposed dialectic between cultural and physiological studies to highlight desirable assumptions for the design and development of an "emotional" prosthesis.

The state of art pointed out that there are many advances in the prosthetic segment, and has shown that the largest fraction is designated to motor functionalities. It confirmed that the crucial point to develop is the insertion of sensors that propose the somatosensory reconfiguration, and five patents addressed to this subject have been detected.

For the neurological aspect, nine desirable requirements for the development of a more sensory prosthesis were noted: compliance, temperature, texture, slip, vibration, pressure, torsion, impedance and position. By the philosophical approach, it is proposed to think of the prosthesis as a garment, with the use of nonconventional form and materials, that approach the object more of the phenomenon like Fashion, than of Medicine.

However, the relation of the living with the world is not purely ontological, not merely poetic and simply physiological. It is therefore necessary that the studied fields complement each other so that the individual can experience both the desired functionality of his or her prosthesis and their cognitive experiences.

Acknowledgments. This work is financed by Project "Deus ex Machina", NORTE01-0145-FEDER-000026, funded by CCDRN, through Sistema de Apoio à Investigação Científica e Tecnológica (Projetos Estruturados I\&D\&I) of Programa Operacional Regional do Norte, from Portugal 2020 and by Project UID/CTM/00264/2019 of 2C2T - Centro de Ciência e Tecnologia Têxtil, funded by National Founds through FCT/MCTES.

\section{References}

1. Magee, R.: Amputation Through the Ages: the Oldest Major Surgical Operation. ANZ J. Surg. 68, 675-678 (1998)

2. Padula, P.A., Friedmann, L.W.: Acquired Amputation and Prostheses Before the Sixteenth Century. Angiology. 38, 133-141 (1987)

3. Kate, J. ten, Smit, G., Breedveld, P.: 3D-printed upper limb prostheses: a review. Disabil. Rehabil. Assist. Technol. 12, 300-314 (2017)

4. Tyler, D.J.: Restoring the human touch: Prosthetics imbued with haptics give their wearers fine motor control and a sense of connection. IEEE Spectr. 53, 28-33 (2016)

5. Coccia, E.: A vida sensível. Cultura e Barbárie Editora, Florianópolis (2010)

6. Chen, E., Eberman, B., Marcus, B.A.: Method and apparatus to create a complex tactile sensation, (1998)

7. Sabolich, J.A., Ortega, G.M., Schwabe, B.G.: System and method for 
providing a sense of feel in a prosthetic or sensory impaired limb, (2002)

8. Herr, H.M.: Mechanisms and methods for designing and manufacturing a mechanical interface between a wearable device and a segment of the human body, (2016)

9. Altobelli, D.E., Perry, N., Christopher, E.: Arm prosthetic device system, (2016)

10. Herr, H.M., Clites, T.R., Maimon, B., Zorzos, A., Carty, M.J., Duval, J.-F.: Method and system for providing proprioceptive feedback and functionality mitigating limb pathology, (2019)

11. Bear, M.F., Connors, B.W., Paradiso, M.A.: Neurociências: Desvendando o Sistema Nervoso. Artmed, Porto Alegre (2008)

12. Van Den Heiligenberg, F.M.Z., Orlov, T., MacDonald, S.N., Duff, E.P., Henderson Slater, D., Beckmann, C.F., Johansen-Berg, H., Culham, J.C., Makin, T.R.: Artificial limb representation in amputees. Brain. 141, 1422$1433(2018)$ 